\title{
Aplastic anemia associated with severe hemorrhagic cystitis following radiotherapy for prostate cancer
}

\author{
TAITO NAKANO, KOUJI IZUMI, AERKEN MAOLAKE, ARIUNBOLD NATSAGDORJI, HIROAKI IWAMOTO, \\ YASUHIDE KITAGAWA, YOSHIFUMI KADONO, HIROYUKI KONAKA, ATSUSHI MIZOKAMI and MIKIO NAMIKI
}

\begin{abstract}
Department of Integrative Cancer Therapy and Urology, Kanazawa University Graduate School of Medical Science, Kanazawa, Ishikawa 920-8641, Japan
\end{abstract}

Received September 9, 2015; Accepted January 28, 2016

DOI: $10.3892 / \mathrm{mco} .2016 .791$

\begin{abstract}
Hemorrhagic cystitis is a rare complication following radiotherapy for intrapelvic cancer types, including cervical cancer, bladder cancer and prostate cancer. The severity of hemorrhagic cystitis is different in each case, although symptoms improve spontaneously in certain cases, and often significant morbidity requiring numerous interventions occurs. Since no treatment strategy exists with high evidences for such severe hemorrhagic cystitis, urologists have difficulty in solving the bleeding and pain, which the patients suffer. Aplastic anemia is a rare blood disorder, with an incidence reported as $2 / 1$ million individuals annually. Patients have a risk of diffuse bleeding for presentation with anemia, thrombocytopenia and neutropenia. The present report presented a case of severe hemorrhagic cystitis remitted successfully by the treatment for underlying aplastic anemia.
\end{abstract}

\section{Introduction}

Hemorrhagic cystitis (HC) is a rare disease that can occur following radiotherapy and chemotherapy, including cyclophosphamide regimens. HC is characterized by diffuse bleeding from the bladder mucosa. By contrast with infectious cystitis, $\mathrm{HC}$ is a potentially deadly complication (1). Treatment for this difficult disease requires a wide range of invasiveness. For example, intravenous, endoscopic and instillation therapy (aluminium hydroxide, magnesium hydroxide aminocaproic acid, alum, silver nitrate solution) (2-5), hyperbaric oxygen therapy and a vascular approach (selective embolization of the internal iliac arteries) may be possible conservative therapies $(6,7)$. Total cystectomy may be a final option for patients

Correspondence to: Dr Kouji Izumi, Department of Integrative Cancer Therapy and Urology, Kanazawa University Graduate School of Medical Science, 13-1 Takara-machi, Kanazawa, Ishikawa 920-8641, Japan

E-mail: azuizu2003@yahoo.co.jp

Key words: hemorrhagic cystitis, radiation cystitis, aplastic anemia, prostate cancer, radiotherapy with refractory $\mathrm{HC}(8)$. However, high risk of perioperative complications and mortality associated with surgery should be taken into consideration. Aplastic anemia (AA) is a rare blood disorder and patients commonly present with pancytopenia, increasing the risk of bleeding complications, which can be fatal if left untreated (9). The present study reported severe $\mathrm{HC}$, which was unable to be managed with several conservative therapies, however, improved by treatment for underlying AA that was diagnosed belatedly.

\section{Case report}

A 70-year-old male was diagnosed to exhibit prostate cancer with cT3aN0M0, initial prostate specific antigen $19.3 \mathrm{ng} / \mathrm{ml}$, and a Gleason score $5+4$. The patient was treated by high dose rate brachytherapy (19 Gy), followed by external beam radiotherapy (EBRT; 46 Gy) for the whole pelvis, with combined androgen blockade (leuprolide acetate and bicalutamide). Following 3 years of radiotherapy, urinary retention and macroscopic hematuria occurred. Ultrasonography revealed bladder tamponade and left hydronephrosis. Abdominal computed tomography and magnetic resonance imaging revealed no specific findings of the cause. As many minor bleedings were observed in the bladder mucosa by cystoscopy, trans-urethral coagulation was performed (Fig. 1). The posterior wall and the urethral triangle in the bladder were edematous, however, neither major bleeding or a cancerous lesion were detected at this time. A long hematoma from the left ureteral orifice was identified and retrograde pyelography revealed a distorted lesion of the left lower ureter. However, urine cytology from the left ureter revealed no malignancy. Biopsy of the bladder mucosa and ureteroscopy to the left ureter revealed no evidence of disease. Finally, radiation-induced HC was clinically diagnosed with no clear evidence of other diseases. As the hematuria was very severe and associated with frequent bladder tamponade and deteriorating anemia, trans-urethral coagulations were performed repeatedly during the next few months. Intravesical instillation of the mixed compound of aluminium hydroxide and magnesium hydroxide was administered, followed by hyperbaric oxygen therapy. Nevertheless, no improvement of clinical symptom was observed. Subsequent bilateral hydronephroses and renal impairment with the serum creatinine level of $8.72 \mathrm{mg} / \mathrm{dl}$ was developed and required the 


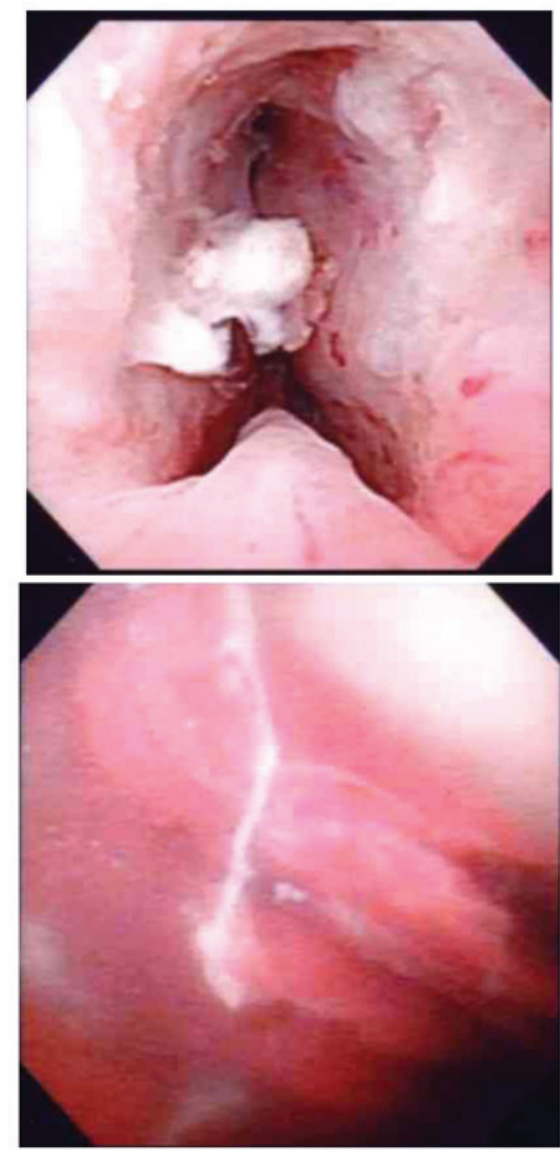

Figure 1. Cystoscopy revealed fur deposition and rough mucosa on the prostatic urethra (upper) and mucosal edema with coagula in the bladder (lower).
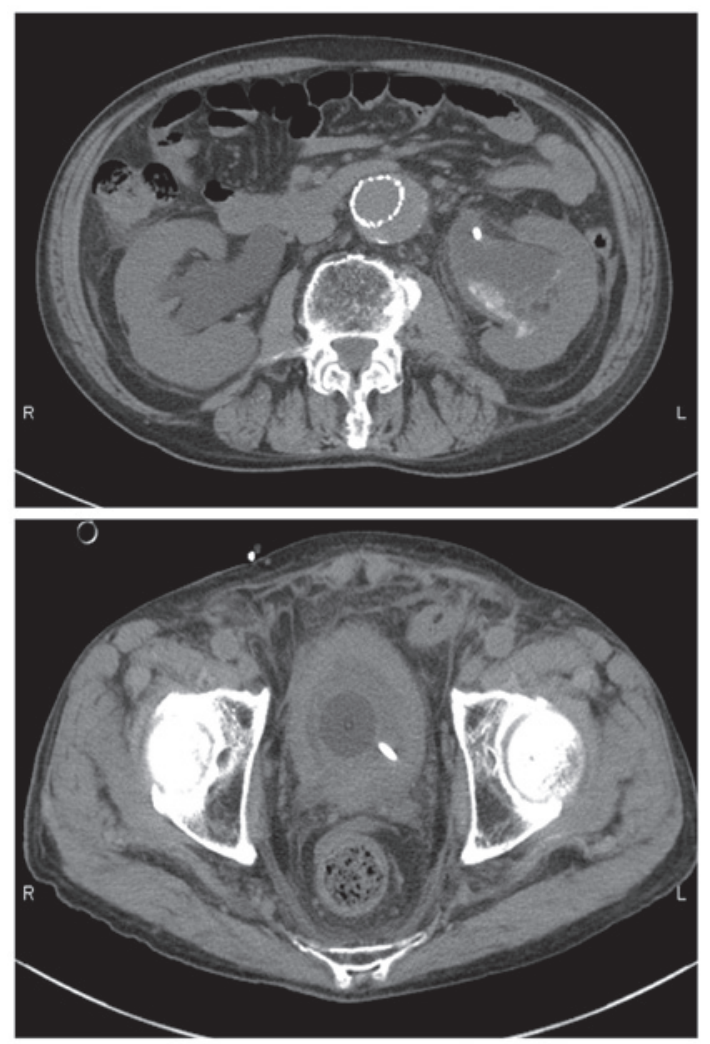

Figure 2. Abdominal computed tomography scan prior to bilateral percutaneous nephrostomies revealed bilateral hydronephroses (upper) and bladder wall thickness with coagula tamponade (lower).

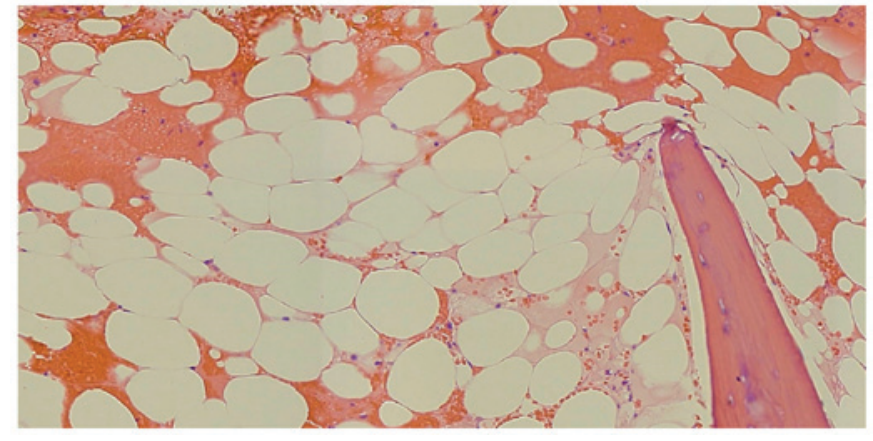

Figure 3. Microscopic appearance of a bone marrow filled with adipose tissue is consistent with aplastic anemia.

induction of hemodialysis as well as bilateral percutaneous nephrostomy (Fig. 2). A blood test revealed pancytopenia and transfusions of red blood cells and platelets were required every other day. When total cystectomy with urinary diversion was considered for this difficult condition, aplastic anemia (AA) was diagnosed by the bone marrow examination (Fig. 3). Administration of cyclosporine and anabolic steroid was initiated by hematologists, and macroscopic hematuria was gradually improved. Subsequently, renal impairment markedly improved to $<1.0 \mathrm{mg} / \mathrm{dl}$ in the serum creatinine level. The incidence of transfusions of red blood cells and platelets was decreased to once a week thereafter.

\section{Discussion}

$\mathrm{HC}$ is defined as a diffuse inflammatory change of the bladder characterized by sustained hematuria or lower urinary tract symptom (10). HC may occur following bone marrow transplantation, peripheral blood stem cell transplantation, radiotherapy to the pelvic organ, chemotherapy using cyclophosphamide, and specific viral infections (10). It is hypothesized that as a result of mucosal edema and inflammation by radiotherapy, telangiectasia and interstitial fibrosis occur, resulting in decreased bladder capacity and compliance (11). Finally, mucosal ischemia, ulceration and bleeding occur. HC can occur generally between 6 months and 10 years after radiotherapy, affecting $\sim 6.5 \%$ of patients following pelvic radiation (11). Leapman et al (12) reported cystoscopy findings of 2,532 males treated with prostate brachytherapy with or without EBRT. Of those, 13 individuals, $(0.51 \%)$ were diagnosed with radiation cystitis, and 8 received combined brachytherapy with EBRT, although the total biological effective dose was similar among these groups (208.6, compared with $200 \mathrm{~Gy} ; \mathrm{P}=0.092$ ). Lawton et al (13) reported that the risk of $\mathrm{HC}$ increases with higher doses of radiation (>70 Gy) and a larger treatment area (13). On the other hand, Fuentes-Raspall et al (14) analyzed 257 patients treated with EBRT for prostate cancer. They reported that late rectal toxicity was associated with the volume irradiated, however, could not correlate with the bladder volume (14). Further studies are required to investigate whether irradiated volume is a risk factor for HC. Treatment of $\mathrm{HC}$ can range from simple bladder irrigation to invasive surgery. For refractory cases to conservative therapy, urinary diversion can be 
considered. However, cystectomy for radiation cystitis is often more difficult. It was reported that the 90-day mortality rate in patients with $\mathrm{HC}$ undergoing a cystectomy was $16 \%$ (15). Cystectomy with urinary diversion is associated with a high rate of perioperative complications and radiation-induced fistula (14). The present study considered this challenging method, however, AA was accidentally diagnosed in the background of HC. On multivariate analysis, increased risk factors of $\mathrm{HC}$ were significantly associated with previous pelvic radiation (10). Although it is unclear whether AA is a risk factor for $\mathrm{HC}$ or not, treatment for AA improved symptoms caused by $\mathrm{HC}$, perhaps as a result of hematopoietic improvement. Close examination in the background of $\mathrm{HC}$ is required in severe and treatment-resistant $\mathrm{HC}$.

\section{References}

1. Haldar S, Dru C and Bhowmick NA: Mechanisms of hemorrhagic cystitis. Am J Clin Exp Urol 2: 199-208, 2014.

2. Kawagoe $K$ and Kawana $T$ : Intravesical instillation of maalox for the treatment of hemorrhagic cystitis. Asia Oceania J Obstet Gynaecol 15: 107-108, 1989.

3. Kawamura N, Kakuta Y, Fukuhara S, Imazu T, Hara T and Yamaguchi S: Successful treatment of hemorrhagic cystitis after radiation therapy with intravesical instillation of aluminium hydroxide gel and magnesium hydroxide: Report of a case. Hinyokika Kiyo 54: 239-241, 2008.

4. Kanwar VS, Jenkins JJ III, Mandrell BN and Furman WL: Aluminum toxicity following intravesical alum irrigation for hemorrhagic cystitis. Med Pediatr Oncol 27: 64-67, 1996.

5. Jerkins GR, Noe HN and Hill DE. An unusual complication of silver nitrate treatment of hemorrhagic cystitis: Case report. J Urol 136: 456-458, 1986.
6. Ribeiro de Oliveira TM, Carmelo Romão AJ, Gamito Guerreiro FM and Matos Lopes TM: Hyperbaric oxygen therapy for refractory radiation-induced hemorrhagic cystitis. Int J Urol 22: 962-966, 2015.

7. Han Y, Wu D, Sun A, Xie Y, Xu J, Zhou J, Zhu X, Wang Z and Ruan C: Selective embolization of the internal iliac arteries for the treatment of severe hemorrhagic cystitis following hematopoietic SCT. Bone Marrow Transplant 41: 881-886, 2008.

8. Linder BJ, Tarrell RF and Boorjian SA: Cystectomy for refractory hemorrhagic cystitis: Contemporary etiology, presentation and outcomes. J Urol 192: 1687-1692, 2014.

9. Willis L, Rexwinkle A, Bryan J and Kadia TM: Recent developments in drug therapy for aplastic anemia. Ann Pharmacother 48: 1469-1478, 2014.

10. Riachy E, Krauel L, Rich BS, McEvoy MP, Honeyman JN, Boulad F, Wolden SL, Herr HW and La Quaglia MP: Risk factors and predictors of severity score and complications of pediatric hemorrhagic cystitis. J Urol 191: 186-192, 2014.

11. Alesawi AM, El-Hakim A, Zorn KC and Saad F: Radiation-induced hemorrhagic cystitis. Curr Opin Support Palliat Care 8: 235-240, 2014.

12. Leapman MS, Stock RG, Stone NN and Hall SJ: Findings at cystoscopy performed for cause after prostate brachytherapy. Urology 83: 1350-1355, 2014.

13. Lawton CA, Won M, Pilepich MV, Asbell SO, Shipley WU, Hanks GE, Cox JD, Perez CA, Sause WT, Doggett SR, et al: Long-term treatment sequelae following external beam irradiation for adenocarcinoma of the prostate: Analysis of RTOG studies 7506 and 7706. Int J Radiat Oncol Biol Phys 21: 935-939, 1991.

14. Fuentes-Raspall R, Inoriza JM, Rosello-Serrano A, Auñón-Sanz C, Garcia-Martin P and Oliu-Isern G: Late rectal and bladder toxicity following radiation therapy for prostate cancer: Predictive factors and treatment results. Rep Pract Oncol Radiother 18: 298-303, 2013.

15. Linder BJ, Boorjian SA, Hudolin T, Cheville JC, Thapa P, Tarrell RF and Frank I: Late recurrence after radical cystectomy: Patterns, risk factors and outcomes. J Urol 191: 1256-1261, 2014. 